\title{
PENSATA
}

\section{MODERNIZACIÓN Y GERENCIA PÚBLICA}

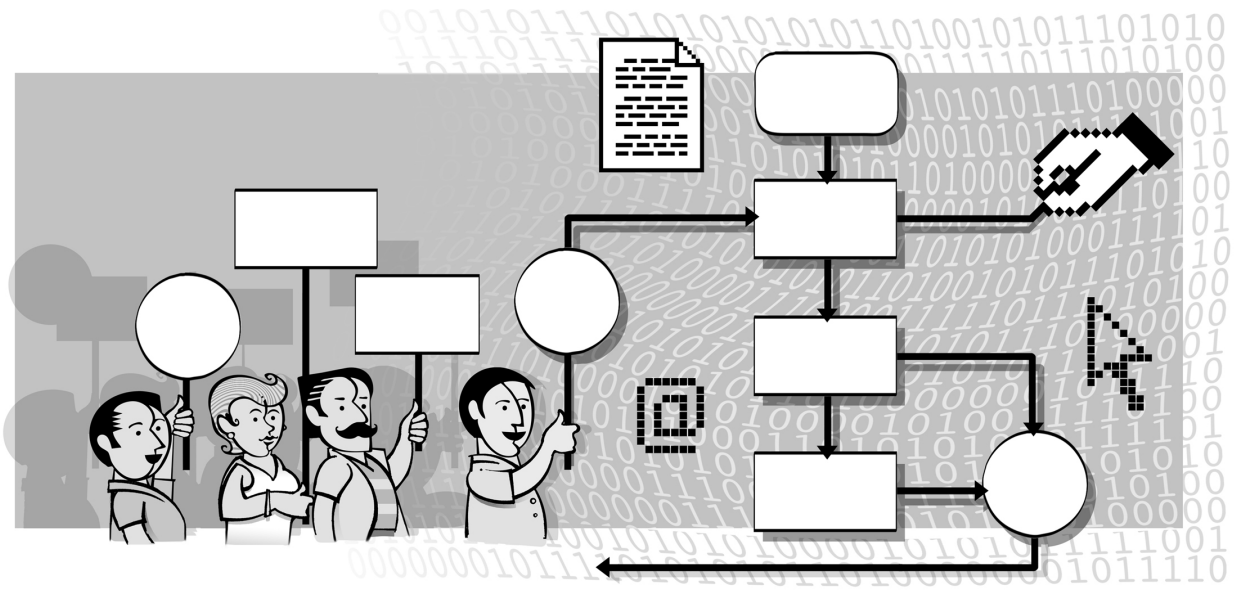

\section{INTRODUCCIÓN}

Si hiciéramos el experimento de conjuntar en un congreso a innovadores del sector público y privado, con experiencia práctica, para debatir modelos de gerencia, el resultado sería una auténtica torre de babel. Hay una inadecuada separación del arte de la gerencia entre sectores públicos y privado; e incluso, un desprecio del privado por el público, en buena parte por los factores que describo en este documento, pero principalmente por un grave problema de percepción y entendimiento de la acción pública.

Un elemento que fortalece la percepción negativa hacia el servicio público es ver a las instituciones públicas como modelo de lo viejo e ineficiente, de lo que no funciona o da resultados y que además genera molestias y costos. Aunque desde hace más de 30 años hay una moda, en algunos casos con resultados muy buenos, por la transformación de la función pública, parece que es un proceso que no ha dado los resultados esperados. Malamente se le califica como un proceso inacabado, cuando debiera ser realmente un proceso permanente, que no tuviera fin.

El entorno global obliga a las organizaciones a estar en constante transformación, como lo señala Michael E. Porter; la única ventaja competitiva real es la capacidad de adelantarse al cambio, ya que la eficacia operativa y la eficiencia no pueden ser una estrategia sostenible, es la rapidez para responder a los cambios competitivos lo que asegura el éxito (PORTER, 1996). ¿Por qué las instituciones públicas no se transforman bajo una visión de adelantarse a los cambios y en cambio sólo lo hacen como reacción? ¿Qué premisas deben orientar la transformación de las instituciones públicas?

Un elemento fundamental para generar innovación y propiciar acciones de transformación

SALVADOR VEGA CASILLAS

salvador.vega@pan.senado.gob.mx Senador del Senado de la Republica . México - MX

\section{ERNESTO GONZÁLEZ CANCINO}

gcancino1@yahoo.com

Oficial Mayor de la Secretaría de

Gobernación de la Republica - México - MX integrales son las crisis. Esta teoría de la crisis como palanca de la transformación debería haber propiciado grandes transformaciones en el sector público, pero no es así. Los escenarios de crisis deberían ser oportunidades de replantear de manera integral a las organizaciones. En el sector privado, han sido las crisis los catalizadores de transformaciones de líneas de negocio, procesos y organizaciones; incluso, nuevos modelos de dirección y administración han surgido de estas coyunturas. Por el contrario, en el sector público, han sido las crisis, factor de distorsión y ambigüedad en la gestión pública (GONZÁLEZ CANCINO, 2012). 
Pero justamente las crisis en las instituciones públicas han tenido un efecto que ha obligado a introducir reformas que gradualmente alcanzan resultados de innovación: la legitimidad. La crisis de la legitimidad de las instituciones públicas han presionado para un repliegue estatal; debido a la poca capacidad del sector público para resolver las demandas sociales; al mismo tiempo que la idea de un gobierno protector y benefactor disminuye y la sociedad reclama más espacios para su autogestión (CABRERO MENDOZA, 1997). Así, surgen nuevas formas de relación sociedad-gobierno, en un ambiente democrático que es obvio, la legitimación de la actuación del gobierno reclama ahora más efectividad, transparencia y participación en la toma de decisiones. Un aparato administrativo incapaz de responder eficazmente a las demandas de la sociedad, dio como resultado el cuestionamiento del Estado como ente obeso, ineficaz e ineficiente. Así, la llamada modernización administrativa surge como una respuesta a la crisis de legitimidad (CABRERO MENDOZA, 1997).

En muchos casos la representación del aparato administrativo como "ente obeso e ineficiente" ha derivado en una transformación hacia el absurdo, el recorte y adelgazamiento; atendiendo solamente los síntomas del problema y no el problema mismo desde su raíz.

Esto significa un grave conflicto para la ciudadanía, que no puede ver los resultados de la acción del gobierno y los servicios que demanda. Así como para el propio gobierno que se ve impedido para dar respuestas eficientes en servicios, enfrentar crisis, poner en marcha medidas de política pública o contracíclicas, debido a la selva normativa, al exceso de estructuras y a la ineficiencia de los procesos.

No se trata de reinventar al gobierno en su estructura y acción, se trata de rediseñar su función para adaptarlo a la nueva dinámica social, para que sea un organismo que conviva en armonía y coordinación con otros organismos, ya sean sociales, públicos o privados; que juegue un papel estratégico en el desarrollo y ante todo, que represente a instituciones de alta evaluación y que el servicio público sea una vocación y profesión de alto respeto, que logre atraer a los mejores ciudadanos.

Antes de emprender un proceso de transformación, conviene hacer una reflexión sobre el objetivo de la misma y las causas que la provocan. Es en el debate entre estos dos conceptos: fin y causa; donde debe estar la claridad que dé sustento a la transformación (VEGA CASILLAS, 2012).

El problema a resolver no es el rediseño de la función pública, sino la valorización de la vocación por el servicio público mediante un aparato de gobierno que genere resultados de calidad para la población, que se adapte a la nueva dinámica social y en consecuencia genere confianza y atraiga talento. Para poder rediseñar, primero hay que responder a las preguntas: ¿para qué? y ¿qué quiero lograr?; antes de definir ¿cómo?

\section{IMPACTO DE LOS CICLOS ECONÓMICOS EN LA ADMINISTRACIÓN PÚBLICA}

Las estructuras de gobierno han tenido que adaptarse a la nueva realidad, derivada de la presión social, adicionando estructuras, procesos y servicios; pero sin evaluar si lo ya existente es necesario (GONZÁLEZ CANCINO, 2012).

Esta necesidad impuesta por la sociedad a los gobiernos para atender nuevas realidades, ha derivado en crecimientos irregulares y amorfos de las instituciones; que fortalece más la percepción de estructuras sin lógica de operación. Por otra parte, los ciclos económicos, cada vez más cortos, ponen al gobierno en periodos de altos ingresos o de escasez de recursos. En los primeros se crean nuevos programas sociales, se fortalecen estructuras y se dan nuevos servicios, en los segundos, los recortes de estructuras y programas son la regla.

Un factor que agrava más el efecto pendular en el tamaño y forma del gobierno, es la cada vez más frecuente "transición de equipos”. Los mandatos de gobierno de décadas han quedado en el pasado, y cada vez es más frecuente que los equipos de alta dirección en el gobierno cambien totalmente, junto con el mandatario, cuando las elecciones así lo determinan; máxime si no se ha logrado consolidar un servicio profesional de carrera en la administración pública.

Así tenemos gobiernos que crecen y se reducen, sin mucha lógica y enfoque; lo que obviamente fortalece más aún la percepción social de instituciones no funcionales e incluso inútiles. Esta mala percepción, sumada a la inexistencia de proyectos de gestión pública de largo plazo alimenta el desprecio social por la vocación del servicio público.

Ante este escenario, en el que las estructuras de gobierno se modifican en tamaño y acción de manera pendular, en que hay cambios de equipos de gestión e incertidumbre en la continuidad de proyectos y en que la percepción social de la gestión de las instituciones públicas es más grave, los gobernantes están obligados a generar transformaciones en el corto plazo.

En muchos casos estas transformaciones producen, desgraciadamente, programas y cambios no sostenibles, que sólo buscan generar una mejora en la percepción y no una transformación de la realidad.

Cito un ejemplo al que hace referencia Transparencia Internacional como un efecto pernicioso que influye en los resultados del Índice de Percepción de la Corrupción: “los golpes espectaculares en algunos países, donde se detiene y encarcela a altos funcionarios por actos de corrupción, genera evaluaciones favorables en la percepción, pero no necesariamente mejoran el desapego social por las prácticas indebidas". Adicionalmente, agregaría que esta mejora en la percepción es momentánea. 
A esto me refiero con los "plafones de modernidad"; a las acciones del gobierno que buscan mejorar la percepción social respecto a su actuar, pero en el fondo, no generan cambios de fondo, ni mejoras sostenibles, ni mucho menos inciden en las actitudes del servidor público.

Un plafón de modernidad es cuando se coloca una nueva fachada al edificio, que lo hace parecer como moderno y eficiente, cuando en realidad, detrás de la escenografía, prevalece lo mismo, sólo que ahora oculto y más costoso.

Si bien, toda transformación de fondo requiere de mejora en la percepción para hacerla sostenible, no debe ser nunca el cambio de la percepción el fin superior, sino un medio para lograr fines de mayor calado.

\section{FUNCIÓN DEL SERVICIO PÚBLICO}

Conviene analizar qué premisas deben estar presentes en el servicio público y comprender la necesidad y alcance de la acción pública.

En muchas de las democracias modernas se discute la llamada "efectividad social de la democracia”, generando un debate sobre si es realmente el mejor modelo de gobernanza pública y cuestionando sus alcances.

Parte de este debate ha alentado que muchos países generen instituciones públicas más allá del sistema de contrapesos entre poderes, con la llamada "moda de las autonomías e instituciones ciudadanas".

La grave desconfianza en las instituciones públicas y en muchos países el desprecio por los partidos políticos, y la cada vez más baja participación en los procesos de elección de gobernantes, ha provocado que legisladores, en respuesta a la crisis de legitimidad, creen cada vez más "instituciones con autonomía" de los poderes centrales, integradas por ciudadanos sin militancia partidista y que, ajenos a intereses políticos, representen a la sociedad.
Este auge de organismos ciudadanos en el gobierno, ajenos a la política y a los partidos, es en el fondo una respuesta social a la desconfianza hacia el servicio público, y la generación de nuevas instituciones públicas que no se sienten gobierno y que ven a la política como un mal. Esto ha provocado que la responsabilidad de la gestión pública se transfiera, que el poder se disperse y debilite y, en consecuencia, que las instituciones públicas se multipliquen, se descoordinen y se conflictúen.

Iniciaba este documento señalando que en toda transformación de la función pública hay que cuestionar ¿para qué?

Generalmente los procesos de transformación surgen por tres causas:

1. Crisis financiera, que provoca la reducción del gasto público y lleva a destinar mayores recursos a programas de atención directa.

2. Crisis funcional, que reclama la atención de nuevas demandas sociales o al menos en un modelo diferente.

3. Crisis de legitimidad. El fuerte cues tionamiento sobre la eficacia de las instituciones públicas provoca este tipo de transformaciones.

Pareciera que las tres causas determinarían de manera directa los fines u objetivos a atender: un mejor gasto administrativo; una mayor cobertura de la acción pública o mayor confianza ciudadana incrementando la efectividad.

Sin embargo, al plantear los modelos de transformación, las causas generalmente se confunden y generan distorsión en los modelos organizacionales. (VEGA CASILLAS, 2012).

No es que se desprecien las tres causas señaladas, pero sí es cuestionable que una transformación se subordine sólo a ellas. El servicio público debe estar siempre subordinado a la generación de mejores oportunidades de desarrollo y no sólo a aspectos intragobierno. Esto genera atención de la crisis, pero sin la solución del problema. En otras palabras, sólo le ponemos un torniquete al herido, para parar la hemorragia, pero nunca atendemos la herida de fondo.

Con estas acotaciones, ahora podemos resumir lo que no debe impulsar una transformación:

a. "Modernidad de plafón", es decir, orientarse más a la percepción que a la transformación de fondo.

b. Transferir responsabilidad y dispersar el poder.

c. Resolver lo inmediato e hipotecar el futuro.

d. Atender la crisis, sin resolver el problema.

e. Transformar hacia adentro sin mirar hacia fuera.

Es necesario que el servicio público esté en una constante transformación, atendiendo la nueva dinámica social, pero no con visión de corto plazo, ni atendiendo las crisis, sin señalar rumbo. Una auténtica revalorización de la acción pública es tan necesaria hoy en día, para alejar fantasmas de la anarquía, del autoritarismo y del desgobierno. La sociedad reclama una acción pública efectiva, que requiere a los mejores ciudadanos para diseñarla e implementarla, los mismos que lidiarán con la posibilidad de que la valuación social inmediata no será positiva.

\section{ESTRUCTURAS, PROCESOS, NORMAS Y ACTITUDES}

La transformación del servicio público es inevitable y tampoco se puede posponer. La demanda social por nuevos servicios públicos, así como la creciente crisis de legitimidad de la acción pública, provocará transformaciones en el ser y hacer del gobierno.

Erróneamente se piensa que el mayor control garantiza mejores resultados; sin 
embargo el control debe entenderse como una política de subsidiariedad. Solamente el necesario, durante el tiempo necesario.

En la función pública vivimos hoy día la dualidad del control. Partimos del principio del servicio público en su regulación, que nos señala que en la acción de gobierno sólo está permitido lo que está autorizado y en la acción privada está todo permitido, con excepción de lo que está prohibido. Así, los funcionarios de gobierno, al ejercer en el sector público debiéramos atenernos solamente a lo escrito y señalado.

Este principio es correcto, sin embargo, la aplicación a "pie juntillas" del mismo, ha generado lo que muchos estudiosos llaman burocratismo, como una acepción peyorativa del burócrata.

La regulación para el gobierno debe señalar qué acción debe ejecutar, pero nunca detallar el cómo de la acción. Es un control de límites estratégicos, pero no de tácticas de actuación. Bajar el concepto de la regulación al detalle, inhibe la creatividad y coarta la libertad del servidor público.

Así, tenemos que regulaciones genéricas, con base en límites muy claros, han generado normas y reglas de acciones, que, buscando mayores eficiencias, provocan lo contrario. La rigidez no puede ser nunca una buena herramienta para la eficiencia.

\section{EL CASO MÉXICO}

En México generamos una transformación de la acción del gobierno, atendiendo estructuras, procesos y normas; pero sobre todo buscando generar actitudes acordes con la misma.

Usamos como eje de la transformación a las normas, obligando a la eliminación de las no funcionales y rompiendo el prejuicio de que a mayor norma mejor gobierno. Tanta innovación como sea posible y sólo las normas que sean necesarias.

La premisa de la transformación fue subordinar la norma al proceso. Partiendo de la realidad. Es decir, primero se identificó la mejor forma de hacer algo (obras públicas, atención médica, expedición de pasaportes, etc.), se diseñó la estructura para ello y finalmente se reguló con un enfoque minimalista, lo estrictamente necesario.

Lo que esto implica es contrario a los modelos de calidad y mejora continua. Podemos decir que se trata de un modelo de rediseño, con injerencia de calidad y mejora, pero al mismo tiempo de reingeniería.

El resultado fue la mayor eliminación de normas en un gobierno, en el mundo. Casi la mitad de las normas fueron eliminadas (15 mil en total) y uno de cada tres trámites (2.500) también se eliminaron; esto implica que los sobrevivientes, para serlo, tendrían que ser transformados. Los trámites son el síntoma, las normas excesivas el mal de fondo.

Menos normas y trámites debe generar mejor estructura (que no necesariamente es menos como fin, pero el efecto sí lo es); pero sobre todo una nueva actitud de agilidad y servicio hacia el ciudadano, visualizando la acción del gobierno, desde la visión del cliente y no del funcionario público.

Una forma de explicar esto es entendiendo un trámite como la acción del gobierno de solicitar documentos al ciudadano, que el mismo gobierno genera, para analizarlos y emitir un nuevo documento. En esta visión, el ciudadano es una bodega de documentos públicos que debe entregar al gobierno, cuando este debiera tenerlos. En un enfoque de transformación de los trámites públicos con visión desde el ciudadano, un gobierno no debería solicitar al ciudadano todo aquel documento que él mismo generó (sin importar qué gobierno lo emite, la fecha, formato o cualquier otra restricción). Este es un ejemplo de una norma basada en límites claros con enfoque ciudadano.

\section{El papel de la tecnología}

De entrada, este cambio en la norma genera la eliminación de múltiples regulaciones sobre trámites y formatos, y también de estructuras. Hoy las tecnologías de la información y comunicación nos permiten generar sistemas para hacer realidad estas transformaciones.

Llegará el momento, en que el único requisito que un ciudadano deberá proporcionar al gobierno para un trámite es su identificación y autorización para el mismo con mecanismos biométricos.

Imaginemos un gobierno donde los documentos en papel no existen, donde la comunicación entre agencias gubernamentales es inmediata y por medios electrónicos, donde las reglas y restricciones son mínimas y estratégicas, donde el costo de operar al gobierno se reduce a casi nada, donde la mayor aportación de los funcionarios es su capacidad intelectual y no la repetición de tareas. Sin lugar a dudas, este modelo de gestión pública, no es un gobierno débil, sino un gobierno fuerte; sí pequeño, pero para ser fuerte no hay que ser grande.

Esta transformación, como ya se ha analizado, puede tener distintas facetas; pero para ser responsable debe tener las siguientes características:

a. De largo plazo. Que no sólo sea para atender una crisis.

b. Sostenible. Que no sólo sea una moda, sino que resista a los vaivenes políticos y económicos.

c. Integral. Que abarque estructuras, procesos, normas y actitudes.

d. Permanente. Que no sea única y acabada, sino que propicie la innovación y cambio continuo.

\section{EL TIEMPO}

Toda transformación debe resistir una paradoja del tiempo; realizarse en el corto plazo pero con efecto en el largo plazo. Las transformaciones de fondo que se realizan en un periodo prolongado terminan en procesos dispersos y desarticulados. Los grandes cambios son "shocks" de intensidad, 
sin tiempo para meditarlo, pero con salidas para corregirlos.

El proceso habitual de la gestión, no considera espacios para la transformación; de hecho se interponen. La solución es crear un proceso acelerado y riguroso, que opere en una vía separada y promueva tanto la velocidad como el compromiso (MILES, 2010).

Pero por otro lado, la transformación deberá dar resultados inmediatos (ganancias rápidas), ya que si no los presenta, su continuidad está en riesgo; hay que recordar que toda organización se resistirá a la transformación, no ver esa negación es el peor error en un proceso de cambio, y el segundo error es querer enfrentarse a esa resistencia.

Los resultados inmediatos deben ser sólo para sostener la transformación, nunca verse como los resultados definitivos y superiores. Una transformación que se precie de serlo, deberá entregar resultados en el largo plazo, no porque se aplacen los mismos, sino que por su trascendencia requieren de ese periodo.

Resolver esta paradoja, actuar de inmediato, entregar ganancias rápidas pero ver efectos en el largo plazo, es la primera condición para generar cambios estructurales en el servicio público.

\section{La sostenibilidad}

La segunda condición se refiere a la sostenibilidad de la transformación. En todo cambio siempre existirán los agentes de la regresión. El anhelo por el pasado, por no cambiar, no debe entenderse como un rechazo a la transformación.

La resistencia al cambio es un fenómeno que existe y no se debe despreciar; pero la forma errónea de atenderla es confrontarla. Todo proyecto de transformación debe garantizar su permanencia en el tiempo, no hacerlo sería irresponsable; ya que el costo inicial de una transformación (deberíamos llamarle inversión, pero algunos críticos de la gerencia pública cuestionan el térmi- no, por lo que no entraremos en esa polémica) es alto, y el abandono de un proyecto de transformación antes de ver resultados sólo provocaría, además de los costos sin logros, menguar el espíritu de transformación.

El centro mundial de innovación de Procter \& Gamble señala que solamente el $15 \%$ de sus proyectos tiene algún resultado, y se evalúa como exitoso sólo el $2 \%$ de los mismos. Lo único seguro en un proceso de transformación es que tendrá errores, actuar de manera responsable para sostener el cambio implica reconocer el error y reaccionar ante el mismo, pero no con abandono, sino con replanteamiento.

En el sector público los vaivenes políticos son un alto riesgo para las innovaciones. Es muy común que proyectos de transformación sin estar aún maduros sean cuestionados por los nuevos directivos. Generalmente los ciclos de proyectos de cambio son mucho mayores a los ciclos de gerencia en la administración pública.

Para ello, se recomiendan cuatro medidas para garantizar la sostenibilidad de los proyectos de transformación:

a. Generar ganancias rápidas que logren darle sostenimiento al proyecto ante dudas, pero asegurar que esas ganancias rápidas no sean el fin del proyecto, sino un medio de subsistencia.

b. Conseguir aliados externos al proyecto, cuya opinión sea de peso ante cualquier duda que genere el estilo de gerencia. Éstos no deben ser vistos como aliados de tu gestión, sino de un proyecto.

c. Involucrar a los servidores públicos que lo implementan. Muchos proyectos de transformación han sobrevivido a modas de gobierno porque quienes lo implementan están convencidos de su resultado y no lo ven como un proyecto de un gobernante, sino como su proyecto de vida. d. Establecer candados legales para evitar regresiones, pero cuidar que esos candados sean estratégicos y no tácticos e incorporar sólo los estrictamente necesarios. Pueden ser decretos y acuerdos al más alto nivel, así como modificaciones legislativas.

\section{La integralidad}

Ya he abordado la transformación que hicimos en México, tomando como base la eliminación de normas y trámites. Pero esta transformación requirió la suma de transformaciones no sólo en normas y procesos, sino en estructuras y actitudes.

Una transformación que se precie de serlo, debe abordar estos cuatro aspectos; uno de ellos debe ser el eje de la misma.

Para el caso México iniciamos el proceso transformador con el lanzamiento del “Concurso para identificar el trámite más inútil”, en el que convocamos a los ciudadanos a decirle al gobierno qué hace mal, señalando los trámites públicos ineficientes e inútiles y otorgando un premio al ciudadano que a juicio de un jurado ajeno al gobierno, detectara en su experiencia el "trámite más inútil" del gobierno.

Este concurso generó un "shock" en el gobierno. Generalmente los gobiernos señalan e informan lo que a su juicio se hace bien; pero en este caso preguntamos a nuestros "clientes" qué hacemos mal. El premio al ciudadano fue entregado por el Presidente de la República con la presencia del Titular de la Institución que prestaba el evaluado por la sociedad como un "trámite inútil".

Esta primera evaluación de lo que no es útil, dio pie a la segunda etapa: eliminar lo que no funciona. Para ello se inició el proceso de regulación base cero.

En este proyecto el Presidente de la República instruyó a sus funcionarios para que en un plazo corto (recordemos que las transformaciones que se dan plazos largos no funcionan) de tres meses inventariaran 
todas las normas y señalaran cuáles debieran eliminarse por no ser necesarias. El resultado de ese primer ejercicio dio una importante reducción de normas.

La transformación no sólo consistía en quitar paja, sino en rehacer las cosas para generar mejores resultados. Por ello, teniendo el inventario de normas, con la precisión de cuáles se habrían de eliminar, la instrucción Presidencial cambió: “No me justifiquen cuáles hay que eliminar, sino cuáles deben prevalecer; como si fuera una evacuación por un desastre natural, sólo dejen lo estrictamente necesario". Nuevamente se otorgó un plazo breve, de un mes, para justificar lo que debiera prevalecer, eliminando todo aquello que en ese plazo no fue debidamente validado.

El resultado: una de cada dos normas fueron eliminadas y uno de cada tres trámites, siendo el mayor ejercicio de desregulación que la OCDE haya registrado en el mundo. Además todo en un plazo corto.

Esta reducción a lo estrictamente necesario, se acompañó de un rediseño de procesos. Una falsa idea en el gobierno es que los procesos deben adecuarse a la norma, ya que ésta debe prevalecer, cuando en realidad una norma tiene como fin garantizar que un proceso sea eficiente y dé resultados. Para ello, el proceso de revisión de normas en los aspectos administrativos del gobierno no consistió sólo en eso, revisar normas, sino en primer lugar rediseñar procesos, y una vez que éstos fueron validados como eficientes y eficaces por quienes los operan y quienes son sus clientes, se procedió a redactar la norma desde "cero"; es decir sin analizar, ver o corregir la existente. Es decir, se subordinó la norma al proceso.

Esto significa un rompimiento del modelo "pontificador", en el que los funcionarios encargados de emitir las normas señalan las prácticas y las establecen como obligatorias. Este modelo asume que el funcionario normado carece de capacidad para proponer procesos que den mejor resultado, que los imprevistos y las circunstancia deben adaptarse a la norma emitida y que el apego a la norma es el fin superior, incluso por encima del fin que persigue la existencia de la norma. Y si la norma no se apega a la realidad, peor para la realidad.

El cambio en las normas y en los procesos no está terminado si no afecta a las estructuras. Tal vez, este sea el de mayor trabajo en las instituciones (y no sólo me refiero a las públicas); porque afecta en buena parte la actividad, responsabilidades e ingresos de las personas.

Las principales políticas de transformación en las estructuras organizacionales han sido la respuesta a recortes de estructuras y la exigencia de atender nuevos proyectos; pero son pocos los casos que parten de un análisis integral sobre qué estructura organizacional debemos tener para atender a la dinámica presente y futura.

Un miedo grave en una transformación es a ser más pequeños, con la falsa idea de que el tamaño implica poder. En la Secretaría de la Función Pública ampliamos acciones, competencias y resultados con una reducción en la plantilla de personal y en el presupuesto de más de 30\%. Es posible hacer más con menos, si sabemos qué es lo menos que nos otorga más resultados, y no sólo cortamos. No se trata de eliminar, sino de hacer cosas diferentes y de mejor forma.

\section{La cultura}

Si se han cubierto los aspectos anteriores seguramente se tendrá una transformación que genere resultados, pero eso no es todo.

Hemos dicho ya que la única ventaja competitiva hoy día es la capacidad de transformarse y adelantarse a los cambios. Por lo que toda empresa de cambio debe ir a las actitudes y valores de los integrantes de la organización.

La falsa idea de las transformaciones acabadas debe desaparecer de las instituciones públicas, no porque no sea importante poner metas y resultados en la línea del tiempo, sino porque todo proceso de transformación debe dar pie a nuevos cambios.
Los incentivos para la transformación son el elemento clave. Si una organización tiene miedo o rechaza la transformación, o la ve como un aspecto aislado y que se ordena desde la alta dirección, ésta sólo se generará si los dirigentes comparten esa visión.

En cambio, organizaciones que premian la innovación, cuyos esquemas de planeación fomentan la participación de sus integrantes y donde la alta dirección genera esquemas de visión estratégica para adelantarse a la dinámica social, devienen en instituciones proactivas.

La ya mencionada "crisis de legitimidad" no es propia de las instituciones públicas. Para Kotter, la falta de legitimidad en los modelos de desarrollo de la alta gerencia es uno de los elementos que propició el desfase de las organizaciones y sus crisis. Muchas empresas que no generaron esquemas de adaptabilidad al entorno y promovieron la innovación no resistieron los cambios en la era global. Los gobiernos no estamos exentos a ese riesgo.

Una organización que valora a la transformación y la innovación, como fuente de resultados, provoca una dinámica de deseo de actualización de su personal. Una organización que ve la actualidad como una necesidad provoca pensamientos de visión de futuro que generan innovaciones, no para adaptarse, sino para adelantarse.

Los gobiernos debemos revisar nuestros esquemas de diseño organizacional, para que el trámite más sencillo en las instituciones públicas sea transformarse, rediseñarse.

\section{EL GATOPARDO Y EL ALEBRIJE}

La novela "El gatopardo", de Giuseppe Tomasi di Lampedusa, nos narra las vivencias de Don Fabrizio Corbera en Sicilia, entre ellas la célebre frase de Tancredi (sobrino de Don Fabrizio), quien le señala con una frase la capacidad de irse adaptando a las circunstancias sin cambiar nada. En la ciencia política el término "gatopardismo" 
o "lampedusiano" ha sido usado para describir cómo los gobiernos inician transformaciones de las estructuras de poder, pero sólo en su superficie, sin tocar el fondo de las mismas, terminando por cambiar todo, pero en esencia siendo lo mismo.

Para ser inmunes al gatopardismo, las transformaciones de la función pública requieren afectar las actitudes, pero no sólo las del servidor público, sino las de la sociedad también.

Otro gran riesgo, además de los cambios superficiales, es la ausencia de una estrategia integral en la administración pública, que provocará que ésta crezca atendiendo a diversas visiones y estrategias sin tener una idea y visión de conjunto. En México existen unos extraños seres multicolores llamados alebrijes, fabricados por los artesanos, que pueden tener cabeza de caballo, fauces de león, garras de águila, cola de perro, etc. Según los sueños e imaginación de sus creadores.

Las administraciones públicas que no tienen una estrategia integral y una visión superior para su evolución y desarrollo, corren el riesgo de terminar siendo como un alebrije ¿para qué sirve un albrije? De adorno. Un adorno que pronto estorbará.

Es necesario que la sociedad valore en mejor forma la función de gobierno, pero ésta sólo puede ser valorada si se transforma. En la medida en que abandonemos ese círculo vicioso, se generarán cambios trascendentes.

Vivir en un esquema de transformación de la burocracia basado en la novela de Lampedusa, sólo incrementará el desprecio por los asuntos públicos, lo que no es sostenible para ninguna sociedad.

Es necesario que los conceptos de nueva gerencia pública se compartan con los conceptos de la gerencia en tiempos del empowerment, y que se generen transformaciones de fondo, integrales (procesos, normas, estructuras y actitudes), que den resultados inmediatos y que se sostengan en el tiempo.

El servicio público debe profesionalizarse, pero la única forma de garantizarlo, es dando resultados de calidad al ciudadano y la única forma de dar resultados de calidad es transformarse como institución pública. El rediseño es la tarea que se debe emprender.

\section{REFERENCIAS}

CASILLAS, S. V. Rediseñando instituciones públicas y políticas de acompañamiento. (C. I. CLAD, Ed.). Cartagena: CLAD, 2012.

CANCINO, E. G. Rediseno de instituciones publicas bajo premisas de eficacia Cartagena: CLAD, 2012.

KPMG. Encuesta de Fraude en México 2012. México: KPMG, 2011.

MENDOZA, C. E. Del administrador al gerente público. México, D.F: INAP, 1997.

MILES, R. H. Accelerating corporate transformations (Don't lose your nerve!). Harvard Business Review, v. 88, n. 1, p. 68-75, 2010.

PORTER, M. "What is Stragegy". Harvard Business Review, n. 6, p. 61-78, 1996.

TRANSPARENCY INTERNATIONAL. Global corruption barometer 2012. Disponible en www. transparency.org. Acceso en 16.08.2013. 